BMJ Open

Diabetes

Research

\& Care

\title{
Stair climbing/descending exercise for a short time decreases blood glucose levels after a meal in people with type 2 diabetes
}

\author{
Hiroto Honda, ${ }^{1,2}$ Makoto Igaki, ${ }^{1}$ Yuki Hatanaka, ${ }^{1}$ Motoaki Komatsu, ${ }^{1}$ \\ Shin-ichiro Tanaka, ${ }^{1}$ Tetsuo Miki, ${ }^{1}$ Taiga Suzuki, ${ }^{2}$ Tetsuo Takaishi, ${ }^{3}$ \\ Tatsuya Hayashi
}

To cite: Honda $\mathrm{H}$, Igaki $\mathrm{M}$, Hatanaka $Y$, et al. Stair climbing/descending exercise for a short time decreases blood glucose levels after a meal in people with type 2 diabetes. BMJ Open Diabetes Research and Care 2016;4:e00232.

doi:10.1136/bmjdrc-2016000232

Received 24 March 2016 Revised 20 June 2016 Accepted 27 June 2016

\section{CrossMark}

\author{
${ }^{1}$ Toyooka Hospital Hidaka \\ Medical Center, Toyooka, \\ Japan \\ ${ }^{2}$ Laboratory of Sports and \\ Exercise Medicine, Graduate \\ School of Human and \\ Environmental Studies, Kyoto \\ University, Kyoto, Japan \\ ${ }^{3}$ Graduate School of Natural \\ Sciences, Nagoya City \\ University, Nagoya, Japan
}

Correspondence to Professor Tatsuya Hayashi; tatsuya@kuhp.kyoto-u.ac.jp

\section{ABSTRACT}

Objective: We examined whether stair climbingdescending exercise (ST-EX), a convenient method to increase physical activity in daily life, for a short period would acutely improve the postprandial blood glucose (BG) response in people with type 2 diabetes (T2D).

Methods: 16 people with T2D (age $65.4 \pm 1.1$ years) participated in 2 separate sessions. After an overnight fast, each participant consumed a test meal and then kept resting for $180 \mathrm{~min}$, except when performing each 3 min bout of ST-EX at 60 and 120 min after the meal (ST-EX session), or kept resting for 180 min (REST session). ST-EX comprised 6 continuous repetitions of climbing to the second floor (21 steps) at a rate of 80 $110 \mathrm{steps} / \mathrm{min}$ followed by walking down slowly to the first floor at a free step rate.

Results: The BG at 60 min after the meal during the ST-EX session (immediately before the first ST-EX) did not differ from that during the REST session, but analysis of variance revealed a significant interaction between time and treatment $(p<0.01)$. The $B G$ at $150 \mathrm{~min}$ after the meal (30 min after the second ST-EX) was significantly lower than that during the REST session $(p<0.01)$. The area under the curve was also $18 \%$ lower during the ST-EX session than during the REST session $(p<0.05)$. The heart rate and blood lactate levels indicated that the actual intensity of ST-EX was 'hard'. In contrast, the rating of perceived exertion (RPE) indicated that the overall intensity of ST-EX was 'moderate' because of decreased RPE scores during descent.

Conclusions: The present findings suggest that performing 3 min ST-EX 60 and 120 min after a meal may be a useful strategy to accelerate the decrease in postprandial BG levels in people with T2D.

\section{INTRODUCTION}

Postprandial hyperglycemia is recognized as an independent risk factor for cardiovascular events, ${ }^{1-3}$ and is highly prevalent throughout the day in people with type 2 diabetes (T2D), even among those with apparently good glycemic control according to their

\section{Key messages}

- Stair climbing-descending exercise (ST-EX) is a convenient method to increase physical activity without any special equipment.

- Lean, moderately active older people with type 2 diabetes performed $3 \mathrm{~min}$ ST-EX 60 and 120 min after a meal.

- ST-EX significantly hastened the decrease in postprandial blood glucose levels.

glycated hemoglobin (HbAlc) level. $^{4}$ Physical exercise has been widely prescribed as part of the treatment of hyperglycemia, and recent studies have shown that highintensity exercise (HIE) effectively improves postprandial glucose metabolism in people with T2D. Gillen et a $\bar{l}$ demonstrated that 10 bouts of $60 \mathrm{~s}$ high-intensity cycling exercise after a meal reduced the postprandial peak of blood glucose (BG) level and the area under the curve (AUC) of BG in people with T2D. Karstoft et al ${ }^{6}$ demonstrated that premeal 1-hour interval walking in people with T2D (repeated cycles of $3 \mathrm{~min}$ of slow and fast walking) decreased the postmeal incremental BG levels. Francois $e t a l^{7}$ examined the effect of six bouts of 1 min highintensity incline walking $(90 \%$ of maximal heart rate) before each meal in individuals with insulin resistance, and found that the postdinner and subsequent 24-hour BG levels were significantly improved. These studies have clearly shown the clinical benefits of HIE for the management of T2D.

On the other hand, the lack of time, the lack of access to an exercise facility and the perceived difficulty in performing exercise are important barriers to regular participation in physical activity in people with T2D. ${ }^{8}$ Thus, it would be desirable to develop a time-saving and non-strenuous way of HIE 
that assures substantial improvements in glycemic control regardless of weather conditions without the need for dedicated exercise equipment. To address this issue, we examined the acute hypoglycemic effect of two separate $3 \mathrm{~min}$ bouts of stair climbing-descending exercise (ST-EX), an easy-to-perform HIE in daily life. One can increase the overall exercise intensity without much effort by alternately climbing and descending stairs on a flight of stairs, because the subjective intensity is alleviated when descending the stairs. ${ }^{9-11}$

\section{METHODS}

Participants

Sixteen Japanese people with T2D but no macrovascular or microvascular complications (13 men and 3 women, under the age of 75), who regularly visited Toyooka Hospital Hidaka Medical Center (Toyooka, Japan), volunteered for this study. Their clinical characteristics were determined in the outpatient clinic within 4 weeks prior to the experiment (table 1). All participants were under medical nutritional therapy (energy intake: 25$30 \mathrm{kcal} / \mathrm{kg}$ body weight/day) and exercise therapy (lowintensity to moderate-intensity aerobic exercise including walking, cycling and/or calisthenics for 20-60 min/ day), which were discontinued on the experimental days. No participants regularly climbed stairs in their daily life. They were taking oral hypoglycemic agents (glimepiride, metformin, and voglibose $(n=2)$; glimepiride and metformin $(n=2)$; voglibose $(n=2)$; alogliptin $(\mathrm{n}=2)$; glimepiride, miglitol, and vildagliptin $(\mathrm{n}=1)$; voglibose, nateglinide, and vildagliptin $(\mathrm{n}=1)$; metformin and voglibose $(\mathrm{n}=1)$; metformin and miglitol $(\mathrm{n}=1)$; voglibose and nateglinide $(\mathrm{n}=1)$; miglitol and alogliptin $(\mathrm{n}=1)$; metformin $(\mathrm{n}=1)$; sitagliptin $(\mathrm{n}=1))$. No participant was taking $\beta$-blockers or antihypertensive drugs that affect heart rate responses to exercise. Written informed consent was obtained from all participants before the experiments. The Institutional Review Board of Toyooka Hospital Hidaka Medical Center approved the study protocol.

\section{Table 1 Characteristics of study participants}

\begin{tabular}{lc}
\hline Age (years) & $\mathbf{6 5 . 4 \pm 1 . 1}$ \\
\hline Body mass index $\left(\mathrm{kg} / \mathrm{m}^{2}\right)$ & $23.6 \pm 0.7$ \\
Duration of diabetes (years) & $11.3 \pm 1.5$ \\
HbA1c $(\%)$ & $6.9 \pm 0.1$ \\
$\mathrm{HbA} 1 \mathrm{c}(\mathrm{mmol} / \mathrm{mol})$ & $52 \pm 1$ \\
Serum total cholesterol $(\mathrm{mmol} / \mathrm{L})$ & $5.0 \pm 0.1$ \\
Serum triglycerides $(\mathrm{mmol} / \mathrm{L})$ & $1.6 \pm 0.2$ \\
Serum HDL-cholesterol $(\mathrm{mmol} / \mathrm{L})$ & $1.6 \pm 0.1$ \\
Serum LDL-cholesterol $(\mathrm{mmol} / \mathrm{L})$ & $3.1 \pm 0.1$ \\
\hline Values are the mean $\pm S E . ~ N=16$. \\
HbA1c, glycated hemoglobin; HDL, high-density lipoprotein; LDL, \\
low-density lipoprotein.
\end{tabular}

\section{Experimental protocols and analytical methods}

This study was a cross-over design with allocation to two different interventions in random order. On separate days with an interval of 1-2 weeks, after an overnight fast from 21:00 the participants consumed a test meal for breakfast (E460F18; Kewpie, Tokyo, Japan) consisting of $19 \mathrm{~g}$ crackers, $62 \mathrm{~g}$ pudding, and $280 \mathrm{~g}$ chicken cream stew (56.5 g carbohydrate, $18 \mathrm{~g}$ protein, $18 \mathrm{~g}$ fat, $460 \mathrm{kcal}$ ) in 10 15 min between 7:00 and 8:00. The participants took medications including any oral hypoglycemic agents before or after breakfast in their usual way, as prescribed by their physicians. Then the participants sat on a chair for $180 \mathrm{~min}$ (REST session) or sat on a chair for $180 \mathrm{~min}$ except when they performed an $\sim 3$ min bout of ST-EX 60 and $120 \mathrm{~min}$ after the meal (ST-EX session). Each bout of ST-EX comprised six repetitions of climbing and descending stairs. The participants climbed to the second floor of the clinic (21 steps, each $17 \mathrm{~cm}$ in height) at a rate of 80 110 steps/min, made a turn at the top of the flight, and then slowly walked back down the stairs to the first floor at a free step rate. The participants made a turn at the bottom of the flight, and repeated stair climbing and descending for a total of six times without rest. Their heart rate was recorded using a Polar Accurex Plus monitor (Polar Electro, Kempele, Finland). Borg's ratings of perceived exertion (RPE) scores ${ }^{12}$ were recorded immediately after the first and second ST-EX.

For the measurement of glucose, lactate, $\mathrm{C}$ peptide, and non-esterified fatty acid (NEFA), capillary blood samples $(50-60 \mu \mathrm{L})$ were collected from a fingertip before $(0 \mathrm{~min})$ and 60 (immediately before the first ST-EX), 90, 120 (immediately before the second ST-EX), 150 , and $180 \mathrm{~min}$ after the meal. Capillary blood samples were also collected after the first and second ST-EX for the measurement of lactate. Glucose and lactate concentrations were measured using a glucose analyzer (Glutest Ace; Arkray, Kyoto, Japan) and a lactate analyzer (Lactate Pro; Arkray), respectively. The blood samples were then centrifuged, and plasma was collected and stored at $-20^{\circ} \mathrm{C}$ until $\mathrm{C}$ peptide and NEFA concentrations were measured using Ultrasensitive $\mathrm{C}$ peptide ELISA (Mercodia, Uppsala, Sweden) and LabAssay NEFA (Wako, Osaka, Japan), respectively.

\section{Statistics}

All values are reported as the mean $\pm \mathrm{SE}$. The time-course changes of BG, $\mathrm{C}$ peptide, and NEFA were analyzed with two-way repeated measures analysis of variance (ANOVA). For BG and C peptide levels, post hoc analysis was then performed with Tukey's test to assess differences between each data point. Differences in the AUCs between the two sessions and parameters in table 2 were analyzed using paired Student's t-test. The AUCs were calculated using the trapezoid method as follows: $0.5 \times \delta_{(60)} \times 60 \mathrm{~min}+\left(0.5 \times \delta_{(60)}+\delta_{(90)}+\delta_{(120)}+\delta_{(150)}+0.5 \times \delta_{(180)}\right) \times$ $30 \mathrm{~min}$, where $\delta_{(\mathrm{n})}$ is the increase from the premeal value (time $=0$ ) at the time point of $\mathrm{n}$ min. Significance was set at $\mathrm{p}<0.05$. 
Table 2 Profile and physical response to ST-EX

\begin{tabular}{|c|c|c|c|}
\hline & $\begin{array}{l}\text { First } \\
\text { ST-EX }\end{array}$ & $\begin{array}{l}\text { Second } \\
\text { ST-EX }\end{array}$ & Mean \\
\hline $\begin{array}{l}\text { Duration of } \\
\text { exercise (s) }\end{array}$ & $174.8 \pm 5.4$ & $173.8 \pm 5.8$ & $174.3 \pm 3.9$ \\
\hline $\begin{array}{l}\text { Step rate } \\
\text { (step/min) }\end{array}$ & $87.8 \pm 2.7$ & $88.5 \pm 2.9$ & $88.2 \pm 2.8$ \\
\hline \multicolumn{4}{|l|}{ Borg RPE scale } \\
\hline RPE-chest & $12.0 \pm 0.5$ & $11.9 \pm 0.5$ & $11.9 \pm 0.4$ \\
\hline RPE-legs & $12.9 \pm 0.3$ & $12.6 \pm 0.4$ & $12.8 \pm 0.2$ \\
\hline$\%$ HRmax & $77.8 \pm 2.0$ & $77.9 \pm 2.1$ & $77.9 \pm 1.4$ \\
\hline \multicolumn{4}{|l|}{ Lactate (mmol/L) } \\
\hline Pre-ST-EX & $1.6 \pm 0.1$ & $2.0 \pm 0.1 \dagger \dagger$ & $1.8 \pm 0.1$ \\
\hline Post-ST-EX & $4.2 \pm 0.3^{\star \star}$ & $4.3 \pm 0.3^{\star \star}$ & $4.2 \pm 0.2^{\star \star}$ \\
\hline \multicolumn{4}{|c|}{$\begin{array}{l}\text { Values are the mean } \pm S E \text {. } \\
t+p<0.01 \text { versus pre-first ST-EX. } \\
{ }_{* \star}^{*} \mathrm{p}<0.01 \text { versus corresponding pre-ST-EX. } \mathrm{N}=16 \text {. } \\
\% \text { HRmax, percentage of age-predicted maximal heart rate; RPE, } \\
\text { rating of perceived exertion; ST-EX, stair climbing-descending } \\
\text { exercise. }\end{array}$} \\
\hline
\end{tabular}

\section{RESULTS}

The BG at $60 \mathrm{~min}$ after the meal during the ST-EX session (immediately before the first ST-EX) did not differ from that during the REST session; however, it decreased more rapidly during the ST-EX session than during the REST session. The ANOVA revealed a significant interaction between time and treatment on BG $(p<0.01$; figure $1 \mathrm{~A})$. The $\mathrm{BG}$ at $150 \mathrm{~min}$ during the ST-EX session (30 min after the second ST-EX) was significantly lower than that during the REST session $(\mathrm{p}<0.01$; figure $1 \mathrm{~A})$. The decreases in $\mathrm{BG}$ were also greater during the ST-EX session than during the REST session at any sampling period $(p<0.05$; figure 2$)$. Furthermore, the AUC for BG (0-180 min) during the ST-EX session was $18 \%$ lower than during the REST session (ST-EX $428.1 \pm 67.0 \mathrm{mmol} / \mathrm{L} \times \mathrm{min}$ vs REST 521.0 $\pm 50.6 \mathrm{mmol} / \mathrm{L} \times \mathrm{min}, \mathrm{p}<0.05)$.

The ANOVA showed a significant interaction between time and treatment on $\mathrm{C}$ peptide $(\mathrm{p}<0.01)$, but there was no significant difference between sessions at any sampling time (figure 1B). The interaction was not significant in NEFA (figure 1C). The AUCs for C peptide and NEFA (0-180 min) were not different between sessions (C peptide: SE-EX $64.5 \pm 7.7 \mathrm{nmol} / \mathrm{L} \times \min$ vs REST $64.8 \pm 6.1 \mathrm{nmol} / \mathrm{L} \times \min$, NEFA: ST-EX $-28.1 \pm 4.2 \mathrm{mmol} /$ $\mathrm{L} \times \min$ vs $\mathrm{REST}-29.9 \pm 6.9 \mathrm{mmol} / \mathrm{L} \times \min )$.

All the participants completed the ST-EX session without adverse clinical manifestations indicating cardiovascular, respiratory, or orthopedic complications. The percentage of age-predicted maximal heart rate $(\%$ HRmax; table 2) indicated that ST-EX was indeed an HIE, because activity is classified as 'hard' when the heart rate is between $70 \%$ and $89 \%$ HRmax. ${ }^{13}$ Moreover, the blood lactate level was robustly increased at the end of ST-EX (table 2). Nevertheless, the participants performed ST-EX without serious symptoms such as dyspnea or leg exhaustion, and the overall extent of
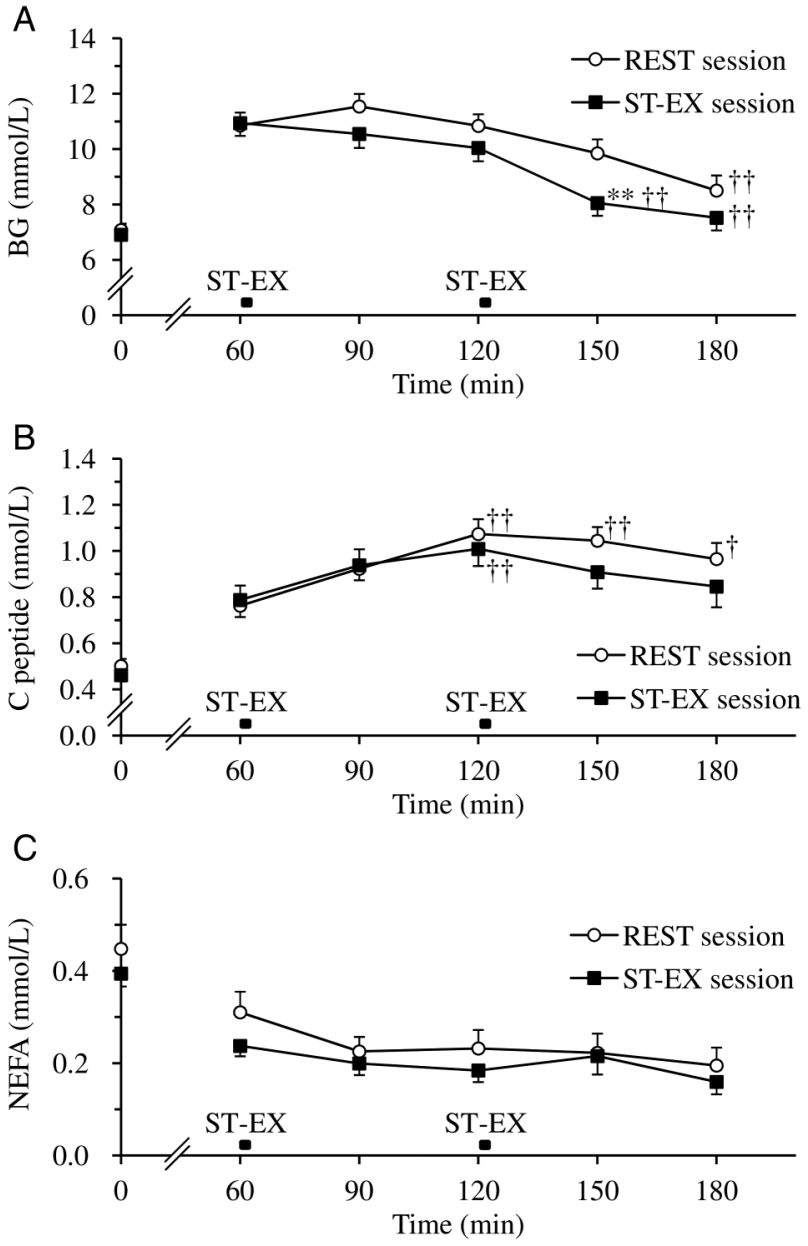

Figure 1 Time-course changes in blood glucose (BG; A), C peptide (B), and non-esterified fatty acid (NEFA; C) levels.

Participants kept resting for 180 min except when performing each 3 min bout of stair climbing-descending exercise (ST-EX) at 60 and 120 min postmeal (ST-EX session), or kept resting for $180 \mathrm{~min}$ (REST session). There were significant interactions between time and intervention on $B G(p<0.01)$ and $C$ peptide $(p<0.01)$, but not NEFA. Values are the mean $\pm S E$. ${ }^{* *} p<0.01$ versus corresponding REST. $\uparrow p<0.05$, $\dagger+\mathrm{p}<0.01$ vs $60 \mathrm{~min} . \mathrm{N}=16$.

physical effort estimated by the RPE for ST-EX was at the 'moderate' level. ${ }^{13}$

\section{DISCUSSION}

We reported previously that a continuous 6 min bout of ST-EX starting $90 \mathrm{~min}$ after a meal accelerated the decrease in postprandial BG levels in people with impaired glucose tolerance (IGT).${ }^{9}$ We also demonstrated that a continuous $6.5 \mathrm{~min}$ bout of ST-EX starting 90 min after ingestion of a carbohydrate solution hastened the decrease in BG levels in people with T2D. ${ }^{11}$ However, we have realised that even the $6-6.5 \mathrm{~min}$ bout of ST-EX is too strenuous for some unfit people to perform regularly in daily life. Therefore, we conducted this study to clarify whether ST-EX for a shorter duration 


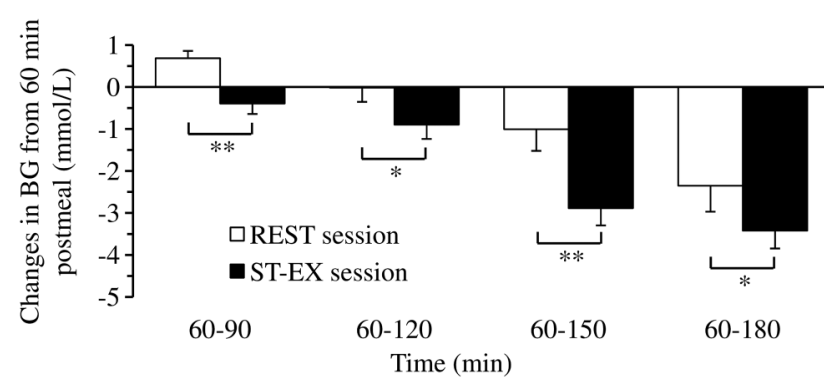

Figure 2 Changes in BG levels from 60 min (immediately before the first stair climbing-descending exercise (ST-EX)) to 180 min postmeal. Participants kept resting for 180 min except when performing each 3 min bout of ST-EX at 60 and $120 \mathrm{~min}$ postmeal (ST-EX session), or kept resting for $180 \mathrm{~min}$ (REST session). Values are the mean $\pm S E$. ${ }^{*} p<0.05,{ }^{* *} p<0.01$ versus corresponding REST. $\mathrm{N}=16$.

( $\sim 3 \mathrm{~min}$ ) would be sufficient to reduce the postprandial response in people with T2D. We employed two separate bouts of ST-EX after a meal, on the basis of our preliminary experiments, indicating that a single $3 \mathrm{~min}$ bout of ST-EX had a limited effect on BG levels (data not shown).

In the present study, we chose the timing for the first ST-EX (60 min after the meal) so as not to increase the BG levels again after exercise. Larsen et $a l^{14} 15$ demonstrated that moderate-intensity to high-intensity cycling exercise during the rapid rising phase of $\mathrm{BG}$ after a meal decreased the BG levels during exercise, but resulted in a rapid rebound after exercise. Similarly, we found in our preliminary experiments that ST-EX during the rapid rising phase of BG (<60 min) induced a rebound in BG levels after exercise (data not shown). As shown in figure 2, the first ST-EX was sufficient to hasten a decrease in the BG level after the meal. We added the second ST-EX (120 min after the meal) to boost this decrease during the declining phase in BG levels. As a result, our protocol clearly maintained a hypoglycemic effect up to $180 \mathrm{~min}$ (figure 2). However, it is possible that addition of ST-EX during the rapid rising phase (eg, at $30 \mathrm{~min}$ ), after the first ST-EX (eg, at $90 \mathrm{~min}$ ) and/or after the second ST-EX (eg, at $150 \mathrm{~min}$ ), might improve postprandial hyperglycemia more significantly than the current protocol.

An acute bout of exercise is a physiologically relevant stimulus to promote glucose uptake in skeletal muscle. Exercise exerts an insulin-like effect on contracting skeletal muscle by stimulating translocation of the glucose transporter 4 (GLUT4) to cell surface membranes independently of insulin. ${ }^{16} 17$ The rate of glucose uptake increases rapidly ( $<5 \mathrm{~min})$ after the initiation of exercise, and depends on the intensity of exercise performed. ${ }^{18} 19$ It has also been shown that the rate of glucose uptake reaches a near-maximal level ( $\sim 80 \%$ of the maximum) within $10 \mathrm{~min}$ after the start of exercise. ${ }^{20}$ Importantly, exercise-induced GLUT4 translocation and glucose uptake in skeletal muscle is intact in people with
T2D. ${ }^{21-23}$ Thus, it seems reasonable to speculate that a 3 min bout of ST-EX is sufficient to substantially increase glucose uptake in working muscles. In addition, the postexercise period has been characterised by enhanced insulin sensitivity in skeletal muscle, which leads to a prolonged increase in insulin-stimulated glucose uptake. $^{24}$ These insulin-dependent and insulin-independent mechanisms might contribute to the acute hypoglycemic effect of ST-EX in people with T2D.

$\mathrm{C}$ peptide and NEFA levels often reflect the intensity and/or duration of exercise. Larsen et $a l^{14}{ }^{15}$ demonstrated that moderate-intensity to high-intensity cycling exercise for $45 \mathrm{~min}$ after a meal acutely blunted the postprandial increases in insulin and $\mathrm{C}$ peptide levels and postprandial decreases in NEFA levels in people with T2D. The reduction in insulin secretion might occur in response to the decreased BG level induced by exercise, as well as from the induction of a counter-regulatory response such as increased sympathetic nervous activity during exercise, ${ }^{25}$ which also causes NEFA release. ${ }^{26}$ In this study, our exercise protocol did not change NEFA levels (figure 1C), suggesting strongly that it does not elicit a clinically unfavorable counter-regulatory effect, and that insulin secretion might have been slightly reduced as a secondary response to the decreased BG levels induced by ST-EX (figure 1A, B).

It is notable that falls on stairs are common among older and/or obese people, particularly those with orthopedic disorders, and that stair descent seems to be more hazardous than stair ascent despite the low exercise intensity during descent. A survey of falls in community-living older people at least 75 years of age demonstrated that $80 \%(20 / 25)$ of falls on stairs occurred during descent. ${ }^{27}$ Aging is accompanied by deteriorations in musculoskeletal capacity for safe stair descent, such as decreased eccentric strength at the knee and ankle. In people with diabetes, visual dysfunction due to retinopathy may also increase the risk of accident. Furthermore, neuropathy is often accompanied by deterioration in sensory, motor, and autonomic nervous systems that may be critical to safe locomotion on stairs. Thus, the use of ST-EX in practice should be individualised with careful consideration of the risk of falling, particularly during descent.

There were some limitations to this study. First, the study participants were lean, maintained optimal HbAlc values, and already under exercise therapy, so the results might not be directly applicable to obese, poorly controlled, or sedentary patients. In particular, physical fitness and body mass index are closely linked in people with T2D: the heaviest people are the least fit, ${ }^{28}$ and thus the decrease in exercise intensity during the descending phase of ST-EX might not be generalised to severely obese patients. Second, no comparison of the exercise intensity between ST-EX and other exercise modalities such as level walking was made. In this regard, we reported previously that a single bout of ST-EX for 6-6.5 min was more effective than level 
walking for the same duration in decreasing postprandial BG in people with IGT $^{9}$ and T2D. ${ }^{11}$ Third, an ST-EX protocol that could attenuate the increase in BG after a meal and blunt the peak postprandial BG, which should be lower than $10 \mathrm{mmol} / \mathrm{L},{ }^{29}$ remains to be determined. Thus, further studies are needed prior to clinical applications of this regimen to a variety of patient populations.

\section{CONCLUSIONS}

In lean, moderately active older people with T2D, 3 min ST-EX starting 60 and $120 \mathrm{~min}$ after a meal hastened the decrease in postprandial BG levels without the need for excessive effort. Although further research is required, ST-EX may be a potentially useful method for increasing physical activity in daily life, with efficient and acute postprandial BG reduction in people with T2D.

Acknowledgements The authors thank the study participants for their time and effort.

Contributors $\mathrm{HH}$ contributed to the study conception and design, acquisition of data, analysis and interpretation of data, and drafting of the manuscript. MI, YH, MK, ST, TM, and TS contributed to the acquisition of data. TT contributed to the study conception and design, analysis and interpretation of data. TH contributed to the study conception and design, acquisition of data, analysis and interpretation of data, and drafting of the manuscript as the guarantor of this work and takes responsibility for the integrity of the data and the accuracy of the data analysis.

Funding This study was supported by JSPS KAKENHI (15K01711) and research grants from the Japanese Council for Science, Technology and Innovation, SIP (project ID: 14533567; funding agency: Bio-oriented Technology Research Advancement Institution, NAR0); Ministry of Agriculture, Forestry and Fisheries, Integration Research for Agriculture and Interdisciplinary Fields (project ID: 14532022; funding agency: Bio-oriented Technology Research Advancement Institution, NARO); and Vascular Disease Research Foundation.

Competing interests None declared.

Ethics approval Toyooka Hospital Hidaka Medical Center.

Provenance and peer review Not commissioned; externally peer reviewed.

Data sharing statement No additional data are available.

Open Access This is an Open Access article distributed in accordance with the Creative Commons Attribution Non Commercial (CC BY-NC 4.0) license, which permits others to distribute, remix, adapt, build upon this work noncommercially, and license their derivative works on different terms, provided the original work is properly cited and the use is non-commercial. See: http:// creativecommons.org/licenses/by-nc/4.0/

\section{REFERENCES}

1. Tominaga $\mathrm{M}$, Eguchi $\mathrm{H}$, Manaka $\mathrm{H}$, et al. Impaired glucose tolerance is a risk factor for cardiovascular disease, but not impaired fasting glucose. The Funagata Diabetes Study. Diabetes Care 1999;22:920-4.

2. DECODE Study Group. Glucose tolerance and cardiovascular mortality: comparison of fasting and 2-hour diagnostic criteria. Arch Intern Med 2001;161:397-405.

3. Nakagami T. Hyperglycaemia and mortality from all causes and from cardiovascular disease in five populations of Asian origin. Diabetologia 2004;47:385-94.
4. Bonora E, Calcaterra F, Lombardi S, et al. Plasma glucose levels throughout the day and $\mathrm{HbA} 1 \mathrm{c}$ interrelationships in type 2 diabetes: implications for treatment and monitoring of metabolic control. Diabetes Care 2001;24:2023-9.

5. Gillen JB, Little JP, Punthakee Z, et al. Acute high-intensity interval exercise reduces the postprandial glucose response and prevalence of hyperglycaemia in patients with type 2 diabetes. Diabetes Obes Metab 2012;14:575-7.

6. Karstoft K, Christensen CS, Pedersen BK, et al. The acute effects of interval- vs continuous-walking exercise on glycemic control in subjects with type 2 diabetes: a crossover, controlled study. J Clin Endocrinol Metab 2014;99:3334-42.

7. Francois ME, Baldi JC, Manning PJ, et al. 'Exercise snacks' before meals: a novel strategy to improve glycaemic control in individuals with insulin resistance. Diabetologia 2014;57:1437-45.

8. Thomas N, Alder E, Leese GP. Barriers to physical activity in patients with diabetes. Postgrad Med J 2004;80:287-91.

9. Takaishi T, Imaeda K, Tanaka T, et al. A short bout of stair climbing-descending exercise attenuates postprandial hyperglycemia in middle-aged males with impaired glucose tolerance. Appl Physiol Nutr Metab 2012:37:193-6.

10. Takaishi $\mathrm{T}$, Ishihara $\mathrm{K}$, Shima $\mathrm{N}$, et al. Health promotion with stair exercise. J Phys Fitness Sports Med 2014;3:173-9.

11. Takaishi T, Hayashi T. Stair climbing/descending exerciseimmediate effect against postprandial hyperglycemia in older people with type 2 diabetes mellitus. Ann Sports Med Res 2015;2:1023.

12. Borg G. Perceived exertion as an indicator of somatic stress. Scand $J$ Rehabil Med 1970;2:92-8.

13. American College of Sports Medicine. American College of Sports Medicine Position Stand. The recommended quantity and quality of exercise for developing and maintaining cardiorespiratory and muscular fitness, and flexibility in healthy adults. Med Sci Sports Exerc 1998;30:975-91.

14. Larsen JJ, Dela F, Kjaer M, et al. The effect of moderate exercise on postprandial glucose homeostasis in NIDDM patients. Diabetologia 1997;40:447-53.

15. Larsen JJ, Dela F, Madsbad S, et al. The effect of intense exercise on postprandial glucose homeostasis in type II diabetic patients. Diabetologia 1999;42:1282-92.

16. Hayashi T, Wojtaszewski JF, Goodyear LJ. Exercise regulation of glucose transport in skeletal muscle. Am J Physiol 1997;273: E1039-51.

17. Jessen N, Goodyear LJ. Contraction signaling to glucose transport in skeletal muscle. J Appl Physiol 2005;99:330-7.

18. Wahren J, Felig P, Ahlborg G, et al. Glucose metabolism during leg exercise in man. $J$ Clin Invest 1971;50:2715-25.

19. Romijn JA, Coyle EF, Sidossis LS, et al. Regulation of endogenous fat and carbohydrate metabolism in relation to exercise intensity and duration. Am J Physiol 1993;265:E380-91.

20. Felig P, Wahren J. Fuel homeostasis in exercise. N Engl J Med 1975;293:1078-84.

21. Minuk HL, Vranic M, Marliss EB, et al. Glucoregulatory and metabolic response to exercise in obese noninsulin-dependent diabetes. Am J Physiol 1981;240:E458-64.

22. Martin IK, Katz A, Wahren J. Splanchnic and muscle metabolism during exercise in NIDDM patients. Am J Physiol 1995;269: E583-90.

23. Kennedy JW, Hirshman MF, Gervino EV, et al. Acute exercise induces GLUT4 translocation in skeletal muscle of normal human subjects and subjects with type 2 diabetes. Diabetes 1999:48:1192-7.

24. Henriksen EJ. Invited review: effects of acute exercise and exercise training on insulin resistance. J Appl Physiol 2002;93:788-96.

25. Kjaer M, Hollenbeck CB, Frey-Hewitt B, et al. Glucoregulation and hormonal responses to maximal exercise in non-insulin-dependent diabetes. J Appl Physiol 1990;68:2067-74.

26. Jensen MD. Fate of fatty acids at rest and during exercise: regulatory mechanisms. Acta Physiol Scand 2003;178:385-90.

27. Tinetti ME, Speechley M, Ginter SF. Risk factors for falls among elderly persons living in the community. N Engl J Med 1988;319:1701-7.

28. Wing RR, Jakicic J, Neiberg R, et al. Fitness, fatness, and cardiovascular risk factors in type 2 diabetes: look ahead study. Med Sci Sports Exerc 2007;39:2107-16.

29. American Diabetes Association. Glycemic targets. Diabetes Care 2015;38(Suppl):S33-40. 


\section{Correction}

Honda H, Igaki M, Hatanaka Y, et al. Stair climbing/descending exercise for a short time decreases blood glucose levels after a meal in participants with type 2 diabetes. BMJ Open Diabetes Research and Care 2016;4:e000232. doi:10.1136/bmjdrc2016-000232.

Some instances of 'Participants' has been changed to 'people' throughout, including the title. The correct title is now 'Stair climbing/descending exercise for a short time decreases blood glucose levels after a meal in people with type 2 diabetes'.

Open Access This is an Open Access article distributed in accordance with the Creative Commons Attribution Non Commercial (CC BY-NC 4.0) license, which permits others to distribute, remix, adapt, build upon this work noncommercially, and license their derivative works on different terms, provided the original work is properly cited and the use is non-commercial. See: http://creativecommons.org/licenses/by-nc/4.0/

BMJ Open Diabetes Research and Care 2016;4:e00232corr1. doi:10.1136/bmjdrc-2016-000232corr1 